\title{
In 2D and 3D Cell Culture Models, Effects of Endothelial Cells on E-cadherin / $\beta$-catenin Expression Levels and Spheroid Sizes in Ishikawa Cells
}

\author{
Mervenur Kalender ${ }^{1,2}$, Muhammet Volkan Bulbul ${ }^{1}$, Bircan Kolbasi ${ }^{1}$, Ilknur \\ Keskin $^{1,2 *}$
}

\begin{abstract}
Objective: Increasing evidence shows that three dimensional cell culture models better reflect the in vivo tumor microenvironment than two dimensional cell culture models. Co-culture models are ideal cell culture models for understanding the communication between cells and the in vivo microenvironment. Changes in expression levels of E-cadherin are closely related to cancer metastasis and progression. $\beta$-catenin mediates cell adhesion of E-cadherin. Endothelial cells are stromal cell components in the tumor microenvironment. It is known that there is little or no expression of E-cadherin in endothelial cells. Methods: In our study, both two-dimensional and three dimensional mono-culture and co-culture models were created using Huvec and Ishikawa cells (endometrial cancer cell lines) to better reflect cell interactions. Spheroids were followed for three days in the three-dimensional cell culture model. E-cadherin and $\beta$-catenin expression levels of two-dimensional and three dimensional mono-culture and co-culture models were measured by immunofluorescence staining. Spheroid images were recorded using a Z-stack. Intensity measurements in both two-dimensional and three-dimensional mono-culture and co-culture models were made using the Image J software. Study groups were evaluated by one-way analysis of variance (One-Way ANOVA). Values of $p$ $<0.05$ were considered statistically significant. Results: The size of the co-culture spheroids was recorded significantly larger than the mono-culture spheroids $(\mathrm{p}<0.0001)$. In two $(\mathrm{p}=0.0175)$ and three dimensional models $(\mathrm{p}<0.0001)$, expression levels of E-cadherin in the mono-culture of Ishikawa cells were recorded significantly higher than in Huvec and co-culture cells. Likewise, while the expression levels of $\beta$-catenin were higher in the mono-culture of Ishikawa cells in two-dimensional models ( $p<0.05$ ), no significant difference was observed in three dimensional models ( $p>$ 0.05). Conclusion: In summary, it has been noted that the expression levels of E-cadherin are significantly reduced in co-cultures of Ishikawa cells with Huvec cells in both two and three dimensions. These results support the idea that endothelial cells may cause changes in endometrial cancer progression by suppressing E-cadherin expression in Ishikawa cells.
\end{abstract}

Keywords: E-Cadherin- Co-culture- Spheroid- $\beta$-Catenin- Immunofluorescence

Asian Pac J Cancer Prev, 23 (1), 39-51

\section{Introduction}

In vitro cell cultures are often used the mechanisms underlying in vivo cell behavior. These behaviors include cell differentiation, migration, growth, and cell mechanics affected by the biochemical and biomechanical environment (Duval et al., 2017). Two-dimensional cell culture techniques are preferred over in vivo studies due to their simplicity and high cell viability (Lee et al., 2008). On the other hand, two dimensional cell cultures have several limitations to understand the communication between cell-matrix and adjacent cells (Kim et al., 2019a). In recent years, some evidence shows that two dimensional cell culture techniques cannot mimic the in vivo responses against the cellular or environmental changes. The morphology of cells that are isolated from the tissue and transferred to two dimensional cell culture conditions is different from the in vivo. Phenotype deprivations are also a negative result of two dimensional culture systems (Kapałczyńska et al., 2018). Because of the implementation of unusual geometric and mechanical constraints to cells, two dimensional cell cultures have only some of the characteristics of normal tissues (Sun et al., 2006).

To understand how tissues are formed, their function, pathophysiology, and their behavior as part of the living 
organism, it is essential to study their three dimensional structures, their mechanical properties, and biochemical microenvironments (Huh et al., 2011). Three dimensional cell culture can mimic the characteristics of cells such as morphology, differentiation, polarity, proliferation rate, gene expression, and genomic profiles, by taking into consideration the important cell-cell and cell-extracellular matrix (ECM) interactions (Koledova, 2017). The cells that do grow in vitro adapt to the new microenvironment, by changes at the genetic, transcriptional and protein levels (Birgersdotter et al., 2005). The three dimensional spheroids is close to that of native tissues due to the formation of both intercellular junctions and interactions between cells and the newly synthetized extracellular matrix (Zurina et al., 2018). Cancer models which are developed in three dimensional cell culture systems are receiving attention, especially due to the lack of well-defined disease models to study metastasis and angiogenesis (Yildiz-Ozturk et al., 2017).

Most tissues in the body consist of more than one cell type. A living tissue constantly interacts with surrounding cells and ECM. This interaction affects the proliferation and differentiation of cells within tissues and organs. Coculture is one of the most studied in vitro method and it has been using to reflect in vivo interactions (Im, 2014).

The tumor microenvironment consists of two different components, cellular and non-cellular. The cellular component involves cancer-associated fibroblasts, tumorassociated endothelial cells, pericytes, immune stromal cells, tumor-associated adipocytes, and cancer stem cells. The extracellular component is comprised of extracellular vesicles such as ECM, soluble factors, and exosomes (Osman et al., 2020).

The role of adhesion molecules includes not only the physical connection between cells and their environment but also the activation of various intracellular signaling pathways. Alterations in both cell-cell and cell-matrix adhesion occur along the multi-step cascade of cancer progression, allowing cancer cells to become more motile, to degrade the extracellular matrix (ECM), to survive in circulation, and to colonize distant metastatic sites (Sousa et al., 2019). The adhesion of each cell is carried out by members of the four main families of adhesion molecules; cadherins, immunoglobulin superfamily (IgSF), selectins, and integrins (Stockmann et al., 2014; Ghasemi et al., 2019; von Lersner et al., 2019). Desmosomes and zonula adherens which are located at the lateral side of a cell, contain cadherin proteins. Cadherins are associated with the preservation of tissue integrity, cell-cell recognition, signaling, intercellular communication, development, and angiogenesis (Schmidt et al., 2011). Epithelial cadherin, a classical type I cadherin, is a key component in the formation of adherence junctions in epithelial tissues. Numerous data over the years have documented the tumor invasion suppressor role of E-cadherin in cancer. Expression of E-cadherin induces low invasive capacity, and formation of functional cell-cell junctions (Sousa et al., 2019). Cadherins, including E-cadherin, are associated with a group of catenin, $\alpha$-catenin and $\beta$-catenin. Catenins act to bind cadherins to microfilaments of the cytoskeleton (Takayama et al., 1996). $\beta$-Catenin is a protooncogene, encoding a cytoskeleton-related protein that is linked to the cytoplasmic extension of E-cadherin in normal epithelial cells mostly in adherence junctions (Pálmer et al., 2001).

In a study showed that expression levels of E-cadherin paralleled the tendency to form spheroids. When expression of E-cadherin was silenced in MCF-7 cells, spheroid was not formed, but overexpression of E-cadherin in SKBR3 cells, a cell line that normally does not form spheroids, caused spheroid formation (Iglesias et al., 2013). In another published study, Du-145 prostate cancer cells have been shown to express small amount of E-cadherin when mono-cultured and a significant increase in E-cadherin expression when cultured with human hepatocytes. It was shown that when Du-145 cells were cultured with fibroblasts, expression of E-cadherin was not observed, thus expression of E-cadherin was induced by hepatocytes (Chao et al., 2012).

Angiogenesis is the process by which new blood vessels are formed. It includes the proliferation, migration, and differentiation of endothelial cells (Liang and Ferrara, 2016). Cancer cells, together with the cells in their microenvironment, stimulate the development of blood vessels by using various tumor angiogenesis mechanisms (Sobierajska et al., 2020). Tumor angiogenesis begins with the activation of specific growth factors that bind to the receptors of endothelial cells (Francavilla et al., 2009).

Based on all this information, in this study, the changes in E-cadherin and $\beta$-catenin expressions in two and three dimensional mono and co-culture models were created with endometrial cancer and endothelial cells, and the effect of these changes on the size of spheroids was examined.

\section{Materials and Methods}

\section{Ethics Committee}

This study was conducted with the approval of Istanbul Medipol University "Non-Interventional Clinical Research Ethics Committee". (Ethics committee approval number: 10840098-604.01.01E.33218).

\section{Cell Culture}

Huvec (Human umbilical vein endothelial cell line-ATCC/CRL-1730) cells were cultured in DMEM F12K (21127022, Gibco) medium containing 10\% FBS (FB-1001/500, Biosera), 1\% streptomycin / penicillin (XC-A4110/100, Biosera) and 0.2\% EGF solution. Ishikawa (endometrial cancer cell line-MERCK/99040201-1VL) cells were cultured in MEM (LM-E1149/500, Biosera) medium containing $10 \%$ FBS, $1 \%$ streptomycin/penicillin, $1 \%$ non-essential amino acid (XC-E1154/100, Biosera). Both cell lines were incubated at $37^{\circ} \mathrm{C}$ in a humidified atmosphere with $5 \% \mathrm{CO}_{2}$. The media of the cells were replaced with fresh medium every two or three days. In both $2 \mathrm{D}$ and $3 \mathrm{D}$ experimental groups, $1 \times 10^{4}$ cells per well for mono-culture models, $5 \times 10^{3}$ Ishikawa cells, and $5 \times 10^{3}$ Huvec cells per well for co-culture models were counted. 
$2 D$ Mono-culture and Co-culture of Huvec and Ishikawa cells

Huvec and Ishikawa cells were seeded $1 \times 10^{4}$ cells per well in 12 well-chamber (81201, Ibidi) for monoculture model. For co-culture model, $5 \times 10^{3}: 5 \times 10^{3}$ Huvec and Ishikawa cells were seeded per well in 12 well-chamber. It was left to grow in an incubator with $5 \% \mathrm{CO}_{2}$ at $37^{\circ} \mathrm{C}$. Huvec and Ishikawa cells were seeded for two-dimensional co-culture applications. In each well of an 8-well chamber, the medium of both cell lines was mixed in a ratio of 1: 1 . The medium of the cells was changed every 2 days during this period.

\section{Preparation of $3 D$ mono-culture and $3 D$ co-culture}

Each well of 96-well plates (VWR-734-2327, Nest) should be coated to prepare a non-adhesive surface. $3 \%$ agarose (BP160-100, Fisher Scientific) was prepared for the coating process. Agarose was dissolved in distilled water by heating in the microwave. Huvec and Ishikawa cells were seeded in 96 well-plate coated with agarose for 3D mono-culture applications. For the mono-culture model, Huvec and Ishikawa cells were seeded at 1x104 cells per well. Huvec and Ishikawa cells were seeded in 96 well-plate coated with agarose for 3D co-culture applications. In each well of a 96 well-plate, the medium of both cell lines was mixed in a ratio of $1: 1$. For the coculture model, 5x103: 5x103 Huvec and Ishikawa cells were seeded per well. It was left to grow in an incubator with $5 \% \mathrm{CO} 2$ at $37{ }^{\circ} \mathrm{C}$. The medium of the cells was changed every 2 days during this period.

\section{Measuring the size of spheroids formed in three dimensional mono-culture and co-culture systems}

Huvec and Ishikawa cells were seeded $1 \times 10^{4}$ cells per well for mono-culture model. From the spheroids created, 30 spheroids were selected for both monoculture and co-culture grouping and followed $(n=30)$. The sizes of Huvec and Ishikawa mono-spheroids were measured 24, 48, and 72 hours after seeding. Huvec and Ishikawa cells were seeded $5 \times 10^{3}$ cells per well for co-culture model. The sizes of Huvec and Ishikawa co-culture spheroids were measured 24, 48, and 72 hours after seeding. The process of measuring the size of all spheroids was recorded using Zeiss Axio inverted microscope and Zen Lite program. All spheroids can't be equal in size in analyzes for immunofluorescence staining. For this reason, the area of the smallest spheroid was determined and the same area was evaluated as common in all spheroids.

Immunofluorescence staining of Huvec and Ishikawa cells prepared in mono-culture and co-culture models in $2 D /$ $3 D$ cell culture

Cells were seeded as described above for two dimensional immunofluorescence staining. The cells in the wells were expected to be confluent for two days. When the spheroids formed for this experiment reached the proper dimensions, they were transferred into an 8-well chamber (80841, Ibidi). Spheroids were incubated with 4\% PFA (P6148, Sigma-Aldrich) diluted in 1X PBS ( LM-S2041/500, Biosera) for 25 minutes at room temperature for fixation. After 4\% PFA was aspirated, it was washed 3 times for 5 minutes with $1 \mathrm{X}$ PBS. The blocking solution was prepared to be $5 \%$ normal goat serum, $0.3 \%$ TritonX (9002-93-1, Sigma-Aldrich) in $1 \mathrm{X}$ PBS. It was incubated for 2 hours at room temperature in a humidified chamber. For the primary antibody, antibodies of rabbit monoclonal $\beta$-Catenin ( D10A8, Cell Signaling) and rabbit monoclonal E-cadherin (24e10, Cell Signaling) were prepared in dilution solution (GTX30932, Genetex) at a ratio of 1: 200. After the blocking solution was aspirated, it was washed with $1 \mathrm{X}$ PBS 3 times for 5 minutes. Primary antibodies were added. It was left to incubate at $+4^{\circ} \mathrm{C}$ for 24 hours. 1X PBS was used as a negative control. To prepare the secondary antibody after 24 hours, goat-anti-rabbit Alexa Fluor 488 (ab150077, Abcam) was prepared in a 1: 200 dilution solution. After the primary antibody was aspirated, it was washed 3 times for 5 minutes with $1 \mathrm{X}$ PBS. A secondary antibody was added to the wells. It was incubated at room temperature in a humidified chamber for 2 hours. At the end of the incubation period, washing was done 3 times for 5 minutes with $1 \mathrm{X}$ PBS. The slide was covered with a coverslip solution containing DAPI (00-4959-52, İnvitrogen). Spheroid images were recorded using a Z-stack on a laser scanning confocal microscope (Zeiss LSM 800). Two dimensional images were recorded in a laser scanning confocal microscope (Zeiss LSM 800).

\section{Statistical Analysis}

Intensity measurements in both two-dimensional and three-dimensional mono-culture and co-culture models were made using the Image J software. In order to ensure optimization, equal area measurements were made in all Z-Stack sections taken from spheroids. Graph Pad Prism 8 statistical program was used for statistical analysis. Study groups were evaluated by one-way analysis of variance (One-Way ANOVA). The differences between the groups were compared with the Tukey test in analyzes where the sample size was equal, and the Bonferroni test in analyzes where the sample was not equal. Values of $p<0.05$ were considered statistically significant.

\section{Results}

Immunofluorescence Staining Images and Statistical Analysis of E-Cadherin in Two Dimensional Mono-Culture and Co-Culture Model of Ishikawa and Huvec Cells

Two dimensional cells were recorded after immunofluorescence staining with E-cadherin antibody (Figure 1a). Alexa Fluor 488 intensity values were measured with Image $\mathrm{J}$ software. Table 1 has shown whether there is a difference between the three groups. A statistically significant difference was found between the Huvec and Ishikawa mono-culture model $(p=0.0175)$. A statistically significant difference was found between Ishikawa mono-culture and co-culture model $(p=0.0175)$ (Figure 1b-c). Post-hoc analysis of different groups is given in Table 2 . 


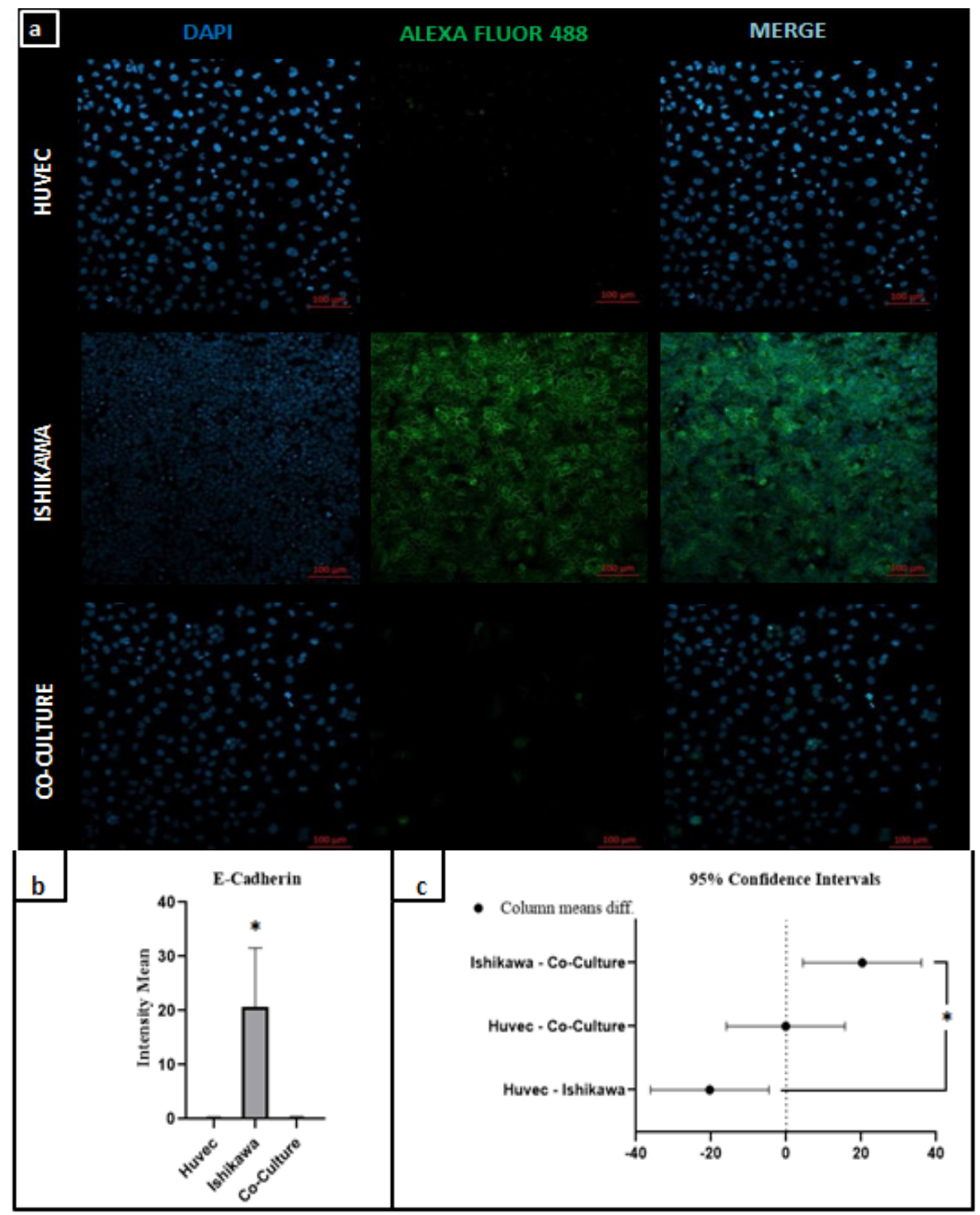

Figure 1. Immunostaining of E-cadherin. Nuclei stained blue with DAPI. (a) Images of Huvec and Ishikawa cells in mono-culture and co-culture models (Scale bar $=100 \mu \mathrm{m})$. (b) Mean E- cadherin expression levels of Huvec and Ishikawa cells in mono-culture and co-culture models. (c) Statistical analysis of mean E-cadherin levels of cells in mono-culture and co- culture models. (Tukey *p<0,05).

Immunofluorescence Staining Images and Statistical Analysis of $\beta$-Catenin in Two-Dimensional Mono-Culture and Co-Culture Model of Ishikawa and Huvec Cells

Two dimensional cells were recorded after immunofluorescence staining with $\beta$-catenin antibody (Figure 2). Alexa Fluor 488 intensity values were measured with Image J software. A significant difference was found between the three groups with the ANOVA test (Table 1). A statistically significant difference was found between the intensity values of Huvec and Ishikawa mono-culture cells $(p=0.0086)$. A statistically significant difference was found between the intensity values of the cells in the Ishikawa mono-culture and co-culture model $(\mathrm{p}=0.0359)$ (Figure $2 \mathrm{~b}-\mathrm{c})$. Table 3 has shown post-hoc analysis of different groups.

Measuring Sizes and Statistical Analysis of Huvec and Ishikawa Spheroids Prepared in Mono-Culture and Co-Culture System

In both systems, the diameter measurements and images of the spheroids formed in 96-well plates were recorded using the Zen Lite program under the microscope 24, 48 and 72 hours after the spheroids started to form (Figures 3-5). The difference between the daily growths of spheroids formed in all three groups was determined using ANOVA (Table3-p valuea). A statistically significant difference was observed between days 1, 2 and 3 of the Huvec spheroids $(p<0.0001)$ (Figure 6a). The mean size of Ishikawa spheroids on day 2 was recorded larger than the other two days $(\mathrm{p}=0.0010)$ (Figure 6b). A statistically significant difference was found between the average sizes of the co-culture spheroids recorded over three days $(p<0.0001)$ (Figure $6 c)$. Whether there was a difference between the groups was first evaluated with the ANOVA test (Table 3-p valueb). In Table 2, spheroid sizes between the groups are compared. The sizes of the co-culture spheroids on the first and second days were measured significantly larger than the Huvec, Ishikawa mono-culture spheroids (Figure 6d-e). The sizes of Huvec mono-spheroids on day 3 were recorded significantly 
DOI:10.31557/APJCP.2022.23.1.39 Effects of Endothelial Cells on E-cadherin / $\beta$-catenin Expression Levels and Spheroid Sizes, 2D-3D Cell Culture

Table 1. Comparison of E-Cadherin Expression of Spheroids in Mono-Culture and Co-Culture System Using ANOVA.

\begin{tabular}{|c|c|c|c|c|}
\hline & Huvec $(\mathrm{N}=3)$ & Ishikawa $(\mathrm{N}=3)$ & Coculture $(\mathrm{N}=3)$ & P value* \\
\hline E-Cadherin (2D) & & & & 0.011 \\
\hline Mean (SD) & $0.28(0.11)$ & $20.66(10.92)$ & $0.39(0.11)$ & \\
\hline Min - Max & $0.16-0.39$ & $13.21-33.19$ & $0.28-0.49$ & \\
\hline$\beta$-Catenin (2D) & & & & 0.009 \\
\hline Mean (SD) & $10.65(3.01)$ & 32.57 (3.99) & $16.73(8.73)$ & \\
\hline \multirow[t]{2}{*}{ Min - Max } & $7.30-13.12$ & $29.44-37.06$ & $8.65-26.00$ & \\
\hline & Huvec $(\mathrm{N}=67)$ & Ishikawa $(\mathrm{N}=46)$ & Coculture $(\mathrm{N}=90)$ & P value* \\
\hline E-Cadherin (3D) & & & & $<0.001$ \\
\hline Mean (SD) & $0.29(0.99)$ & $9.65(21.18)$ & $1.36(5.12)$ & \\
\hline Min - Max & $0.00-4.98$ & $0.00-75.08$ & $0.00-28.46$ & \\
\hline$\beta$-Catenin (3D) & & & & 0.229 \\
\hline Mean (SD) & $8.73(14.42)$ & $10.14(15.07)$ & $19.09(26.18)$ & \\
\hline Min - Max & $0.01-46.14$ & $0.03-47.10$ & $0.30-75.92$ & \\
\hline
\end{tabular}

SD, Standard Deviation; *ANOVA, two sided P.value $<0.05$

smaller than the sizes of both Ishikawa mono-culture and co-culture spheroids $(\mathrm{p}<0.0001)$, and no significant difference was found between the sizes of co-culture and Ishikawa mono-spheroids ( $\mathrm{p}>0.05)$ (Figure 6.f).
Immunofluorescence Staining Images and Statistical Analysis of E-Cadherin Alexa Fluor 488 Intensity Measurements of Huvec, Ishikawa Mono-culture, and Co-culture Spheroids

After Z-stack optical sections of Huvec, Ishikawa

Table 2. Post-Hoc Analysis of Groups with Differences

\begin{tabular}{|c|c|c|c|c|c|}
\hline & Group 1 & Group 2 & $\mathrm{n} 1$ & $\mathrm{n} 2$ & P value* \\
\hline \multirow[t]{3}{*}{ Huvec } & 1.Day & 2.Day & 30 & 30 & $<0.001^{\mathrm{a}}$ \\
\hline & 1.Day & 3.Day & 30 & 30 & $<0.001^{\mathrm{a}}$ \\
\hline & 2.Day & 3.Day & 30 & 30 & $<0.001^{\mathrm{a}}$ \\
\hline \multirow[t]{3}{*}{ Ishikawa } & 1.Day & 2.Day & 30 & 30 & $0.001^{\mathrm{a}}$ \\
\hline & 1.Day & 3.Day & 30 & 30 & $0.235^{\mathrm{a}}$ \\
\hline & 2.Day & 3.Day & 30 & 30 & $<0.001^{\mathrm{a}}$ \\
\hline \multirow[t]{3}{*}{ Coculture } & 1.Day & 2.Day & 30 & 30 & $<0.001^{\mathrm{a}}$ \\
\hline & 1.Day & 3.Day & 30 & 30 & $<0.001^{\mathrm{a}}$ \\
\hline & 2.Day & 3.Day & 30 & 30 & $<0.001^{\mathrm{a}}$ \\
\hline \multirow[t]{3}{*}{ 1.Day } & Huvec & Ishikawa & 30 & 30 & $<0.001^{\mathrm{a}}$ \\
\hline & Huvec & Coculture & 30 & 30 & $<0.001^{\mathrm{a}}$ \\
\hline & Ishikawa & Coculture & 30 & 30 & $<0.001^{\mathrm{a}}$ \\
\hline \multirow[t]{3}{*}{ 2.Day } & Huvec & Ishikawa & 30 & 30 & $0.015^{\mathrm{a}}$ \\
\hline & Huvec & Coculture & 30 & 30 & $<0.001^{\mathrm{a}}$ \\
\hline & Ishikawa & Coculture & 30 & 30 & $0.003^{\mathrm{a}}$ \\
\hline \multirow[t]{3}{*}{ 3.Day } & Huvec & Ishikawa & 30 & 30 & $<0.001^{\mathrm{a}}$ \\
\hline & Huvec & Coculture & 30 & 30 & $<0.001^{\mathrm{a}}$ \\
\hline & Ishikawa & Coculture & 30 & 30 & $0.968^{\mathrm{a}}$ \\
\hline \multirow[t]{3}{*}{ E-Cadherin (2D) } & Huvec & Ishikawa & 3 & 3 & $0.017^{\mathrm{a}}$ \\
\hline & Huvec & Coculture & 3 & 3 & $0.999^{\mathrm{a}}$ \\
\hline & Ishikawa & Coculture & 3 & 3 & $0.017^{\mathrm{a}}$ \\
\hline \multirow[t]{3}{*}{$\beta$-Catenin (2D) } & Huvec & Ishikawa & 3 & 3 & $0.008^{\mathrm{a}}$ \\
\hline & Huvec & Coculture & 3 & 3 & $0.453^{\mathrm{a}}$ \\
\hline & Ishikawa & Coculture & 3 & 3 & $0.035^{\mathrm{a}}$ \\
\hline \multirow[t]{3}{*}{ E-Cadherin (3D) } & Huvec & Ishikawa & 67 & 46 & $<0.001^{\mathrm{b}}$ \\
\hline & Huvec & Coculture & 67 & 90 & $0.999^{\mathrm{b}}$ \\
\hline & Ishikawa & Coculture & 46 & 90 & $<0.001^{\mathrm{b}}$ \\
\hline
\end{tabular}

${ }^{\mathrm{a} T u k e y ~ t e s t ; ~}{ }^{\mathrm{B}}$ Bonferroni test ${ }^{\mathrm{b}}$ 


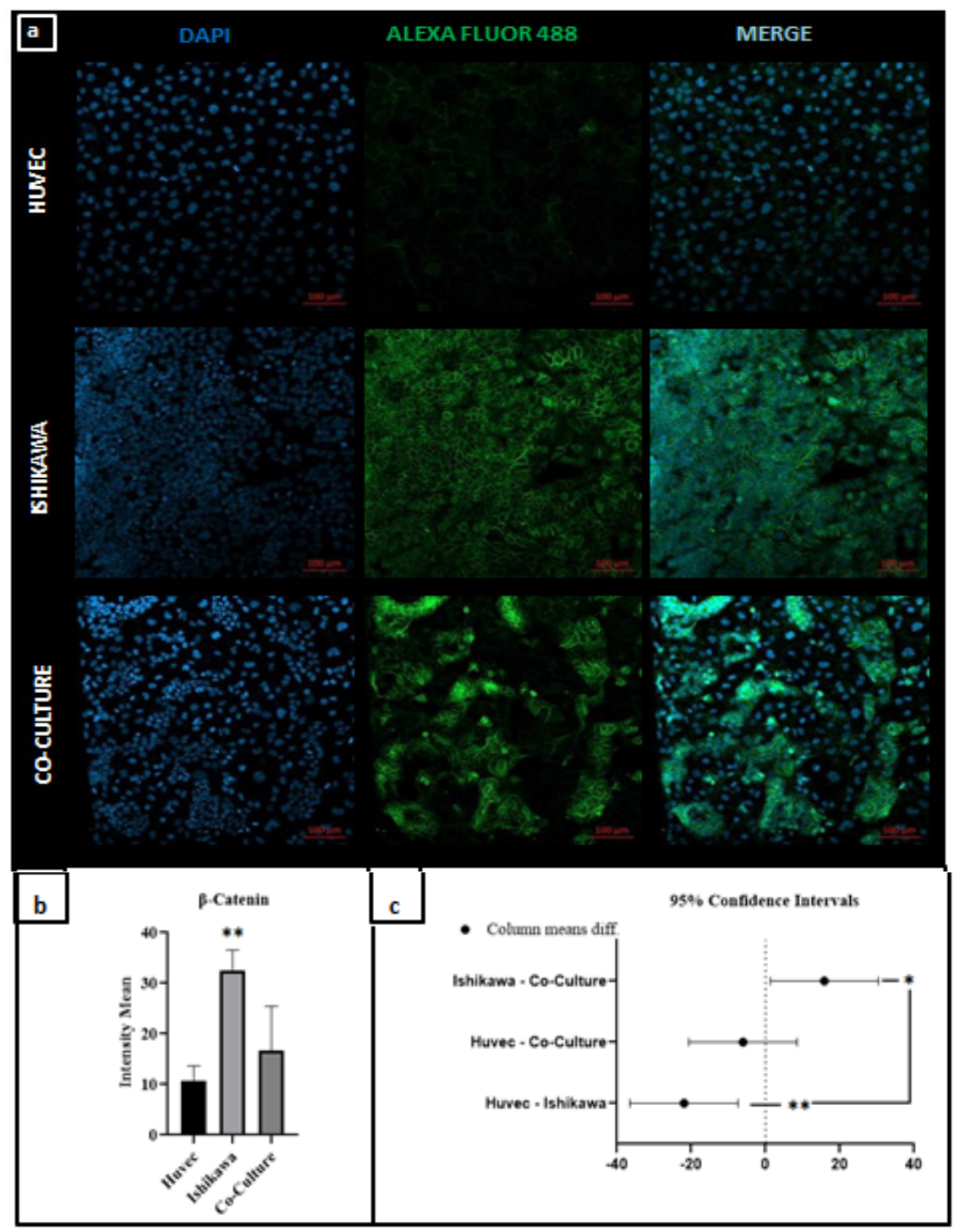

Figure 2. Immunostaining of $\beta$-catenin. Nuclei Stained blue with DAPI. (a) Images of Huvec and Ishikawa cells in mono-culture and co-culture models (Scale bar $=100 \mu \mathrm{m})$. (b) Mean $\beta$ - catenin expression levels of Huvec and Ishikawa cells in mono-culture and co-culture models. (c) Statistical analysis of mean $\beta$-catenin levels of cells in mono-culture and co-culture models. (Tukey $* * p=0,0086, * p=0,0359$ ).

mono-culture, and co-culture spheroids were taken, Alexa Fluor 488 intensity values were measured with Image J software (Figure 7a). Whether there is a difference between the Z-stack optical sections of each group is shown in Table 1. Since there was a significant difference between the expressions values of E-Kader of the groups, post-hoc analysis was performed (Table 2). A statistically significant difference was found between the

Table 3. Comparison of Spheroids Sizes in Mono-Culture and Co-Culture System Using ANOVA.

\begin{tabular}{|c|c|c|c|c|}
\hline & 1. Day $(\mathrm{N}=30)$ & 2. Day $(\mathrm{N}=30)$ & 3. Day $(\mathrm{N}=30)$ & $\mathrm{P}_{\text {value }}$ \\
\hline Huvec & & & & $<0.001$ \\
\hline Mean (SD) & $0.00(0.00)$ & $413.58(34.46)$ & $353.48(30.00)$ & \\
\hline Min - Max & $0.00-0.00$ & $350.60-508.99$ & $295.46-440.04$ & \\
\hline Ishikawa & & & & $<0.001$ \\
\hline Mean (SD) & $415.99(40.38)$ & $452.48(73.95)$ & $430.54(70.82)$ & \\
\hline Min - Max & $365.61-525.36$ & $393.30-736.34$ & $362.46-696.88$ & \\
\hline Coculture & & & & $<0.001$ \\
\hline Mean (SD) & $715.30(54.28)$ & $498.01(41.53)$ & $433.59(34.54)$ & \\
\hline Min - Max & $626.84-880.21$ & $432.25-616.84$ & $371.38-538.74$ & \\
\hline P value ${ }^{b}$ & $<0.001$ & $<0.001$ & $<0.001$ & \\
\hline
\end{tabular}

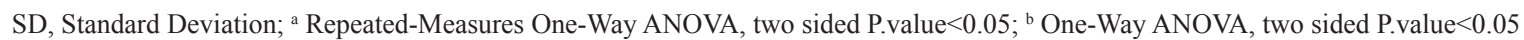




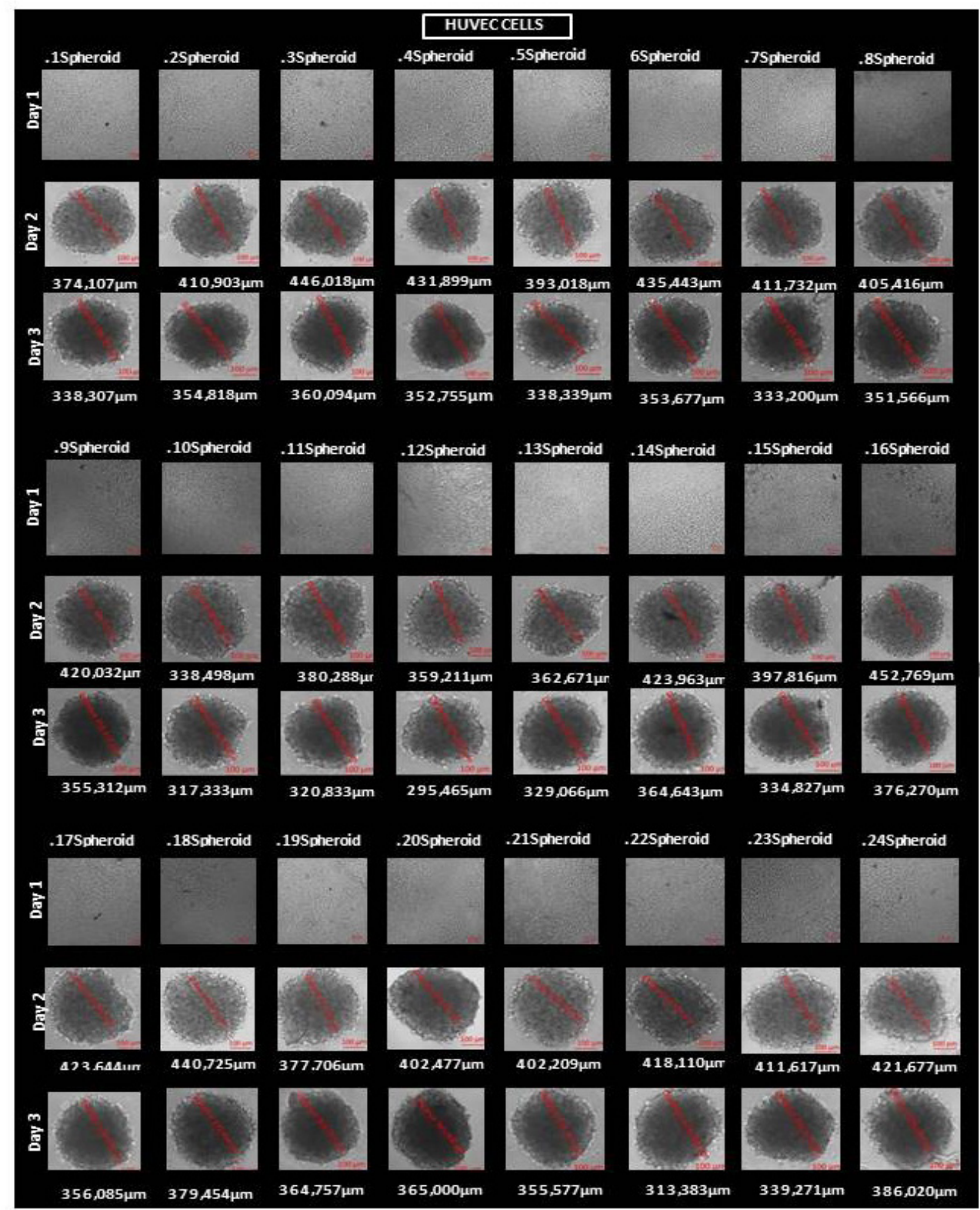

Figure 3. Sizes of Huvec mono-culture Spheroids Measured Over Three Days (Scale bar $=100 \mu \mathrm{m})$.

intensity values of optical sections of Ishikawa and Huvec mono-spheroids $(p<0.0001)$. A statistically significant difference was found between the intensity values of the optical sections of the co-culture spheroid and Ishikawa mono-spheroid $(\mathrm{p}<0.0001)$ (Figure $7 \mathrm{~b}-\mathrm{c}$ ).

Immunofluorescence Staining İmages and Statistical Analysis of $\beta$-Catenin Alexa Fluor 488 Intensity Measurements of Huvec, Ishikawa Mono-culture, and Co-culture Spheroids

After Z-stack optical sections of Huvec, Ishikawa mono-culture, and co-culture spheroids are taken, Alexa Fluor 488 intensity values were measured with Image J software (Fig.8a). No statistically significant difference was found between the intensity values of the optic sections of Huvec, Ishikawa mono-culture and co-culture spheroids ( $\mathrm{p}>0.05)$ (Figure 8b-c).

\section{Discussion}

Standard procedures for compound searching in drug development start with tests based on two dimensional cell culture then continue with animal model tests and clinical trials. Since proper tissue structure and cell-cell interactions disappear under two dimensional culture conditions, the in vivo conditions cannot be fully reflected (Hess et al., 2010; Kim et al., 2019b). Growth on two dimensional surfaces ends up with cell adhesion to the surface and reshaping of the inner cytoskeleton. Such changes have been shown to alter gene expression. Adhesion of the cell to the surface also affects the nuclear shape. The change in nuclear shape can lead to differences in gene expression and protein synthesis. For example, cells cultured in monolayer are considered to be more sensitive to therapeutic agents. It has also been shown that two dimensional cell culture can increase cell proliferation but inhibit cell differentiation due to 


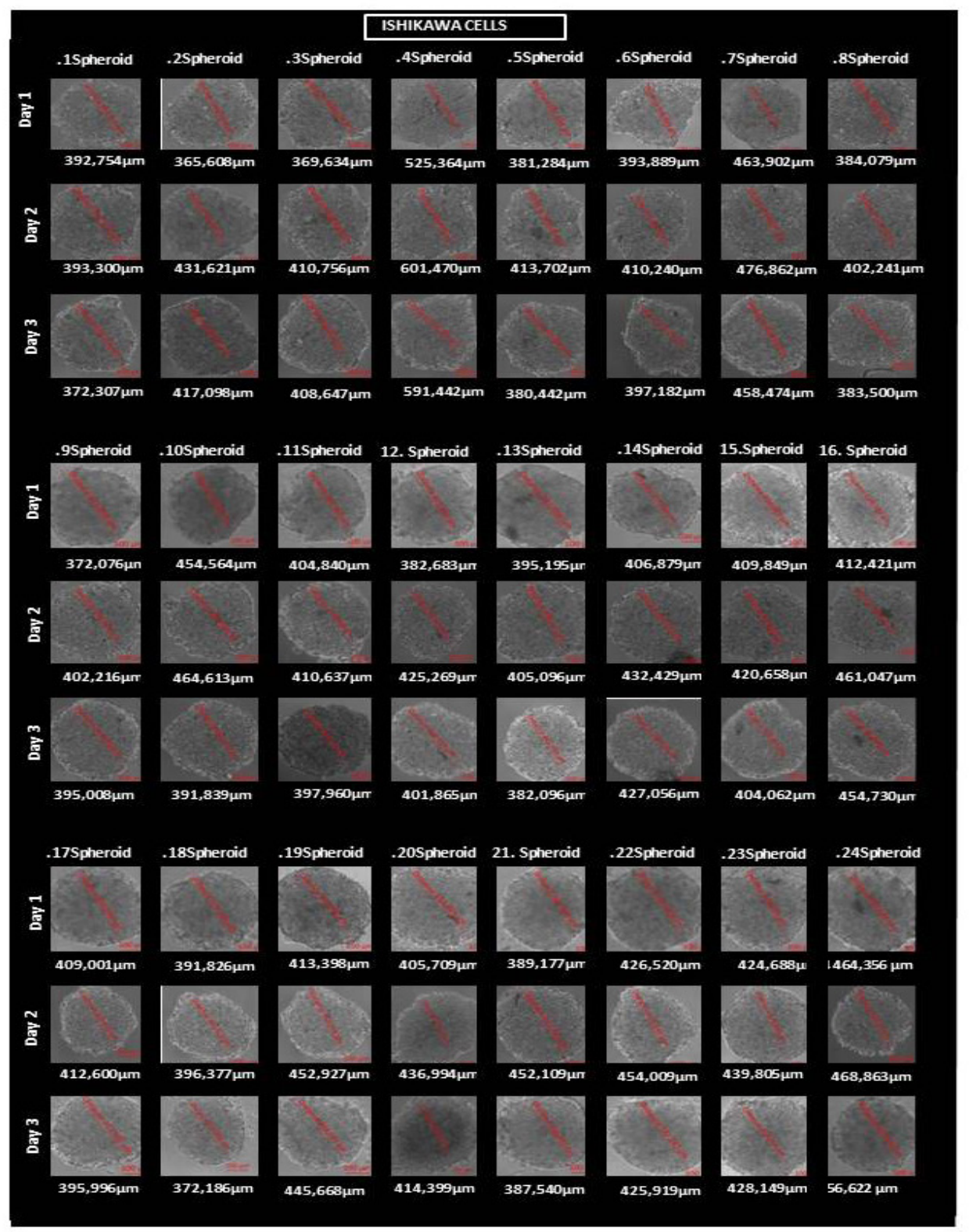

Figure 4. Sizes of Ishikawa Mono-Culture Spheroids Measured Over Three Days $($ Scale bar $=100)$

limited cell interactions (Hess et al., 2010; Knight and Przyborski, 2015).

Solid tumors grow in three dimensional structures in in vivo. Three dimensional cell culture mimics the in vivo environment better than traditional two dimensional cell culture because of its ability to create cell-cell interactions and transform into three dimensional structures, as in vivo. This is supported by the fact that cells grown as $2 \mathrm{D}$ cultures lose some of their natural functional abilities; however these changes can be restored simply by growing cells in 3D again (Breslin and O'Driscoll, 2016).

In co-culture systems two or more different cell types are cultured together in the same vessel or well. Co-cultures are divided into direct co-culture in which they are cultured as a mixed population that allows cell-cell interactions, and indirect co-culture in which two cell populations are separated from each other. Indirect co-cultures techniques cell-cell adhesion and/integrin / notch type signaling cannot be studied
(Marino et al., 2019). In order to examine adhesion molecules we choose to use. In addition, tumor cells were grown with mono-culture and co-culture techniques to understand which a closer model to in vivo tumors is. In a previous study, Panc-1 cells were grown in the mono-culture system and in the co-culture system with fibroblast cells. It was observed that the nuclei of Panc1 cells grown in the mono-culture system were more compact than the nuclei of cells grown in the co-culture system, which means that cancer cells grown in the monoculture system divide faster (Betriu and Semino, 2018).

It was observed of this study that the Huvec cell line does not tend to form spheroids like the endometrial cancer cell line (Ishikawa) and cannot form compact spheroids. It has been noted that the Ishikawa cell line produces more prominent spheroid and compact spheroids. Spheroid formation was not observed 24 hours after the Huvec cells were seeded alone, while Ishikawa mono-culture and co-culture cells were able to form the spheroid 


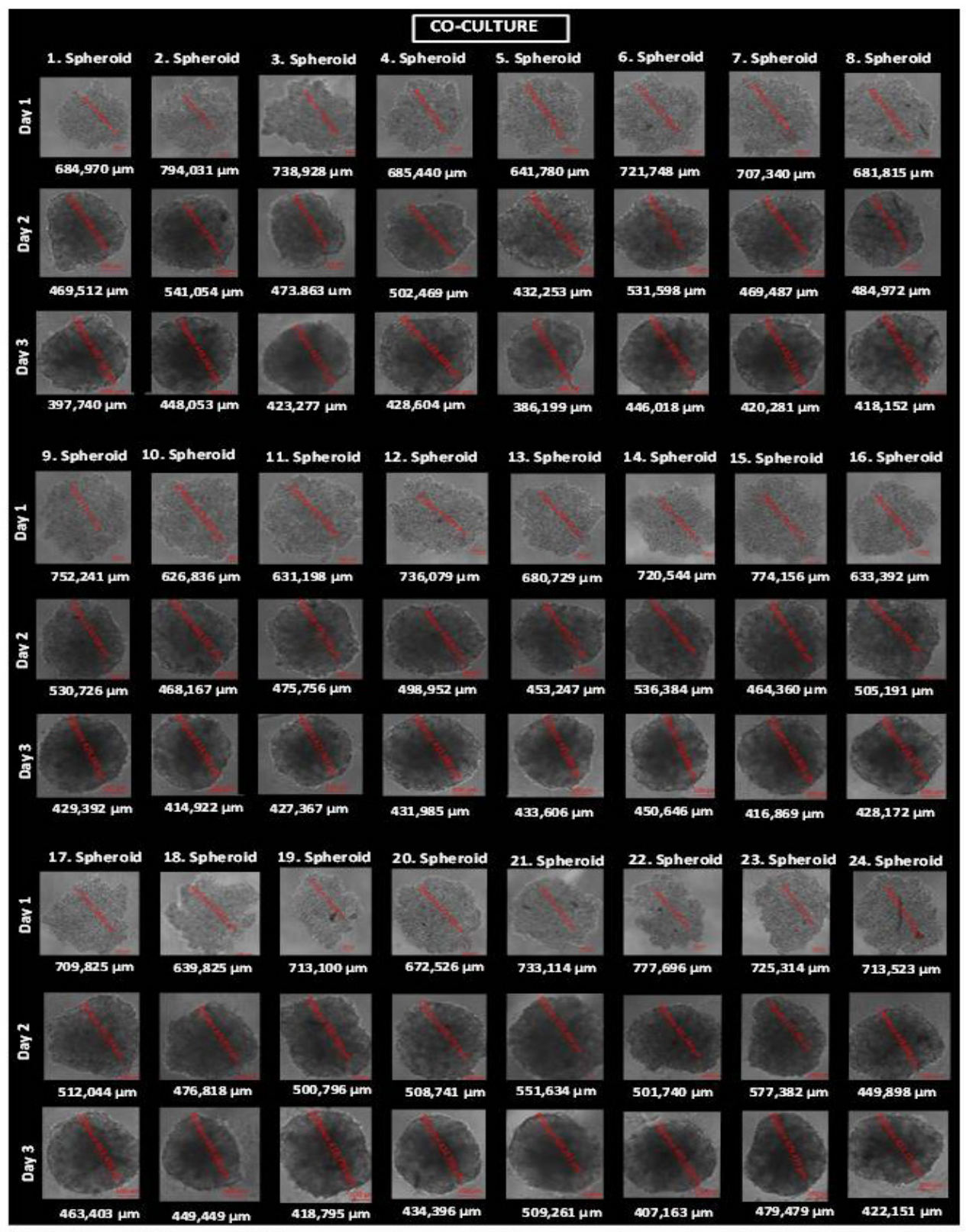

Figure 5. Sizes of Huvec and Ishikawa co-culture Spheroids Measured Over Three Days (Scale bar $=100 \mu \mathrm{m})$.

shape. The co-culture spheroids reached larger sizes than the Huvec and Ishikawa mono-culture spheroids 24 hours after seeding. The co-cultured spheroids showed a statistically significant difference in size on both day 1 and day 2, when compared with other groups. The difference between Ishikawa mono-spheroids on the third day alone is not statistically significant. It is known that endothelial cells in the tumor microenvironment support tumor growth and angiogenesis (Saggioro et al., 2020). One study reported an increase in endothelial cell count in the presence of tumor cells (Khodarev et al., 2003). In addition, tumor angiogenesis is thought to occur with the increase in the presence of endothelial cells (Swaminathan et al., 2019). Another reason for the co-culture spheroids being larger than the other two groups in this study may be that the angiogenesis gradually increases as the number of endothelial cells increases. With the increase of angiogenesis, the essential environment is provided for the growth of tumor cells. As a result of all this, it is consistent with our hypothesis that co-culture spheroids develop better due to the presence of the endothelial cell line and are larger than mono-culture tumor spheroids.

Increasing evidence in recent years has revealed that E-cadherin is associated with undesirable clinicopathological parameters in many human cancers such as breast, stomach, and lung (Zheng et al., 2015). In previous studies with various cancer cell lines, spheroid formation has been associated with increased expression of E-cadherin (Lin and Chang, 2008). However, when cancer cells are co-cultured with stromal cells, expression of E-cadherin has been shown to be reduced (Froeling et al., 2009). In our study, the expression of E-cadherin in Ishikawa mono-spheroids was significantly higher than the other two groups. Also, the expressions of E-cadherin of co-culture spheroids which we generated with Huvec and Ishikawa cell lines were significantly lower than Ishikawa mono-spheroids. It is accepted that that in endothelial cells E-cadherin expression is low, besides that in some 


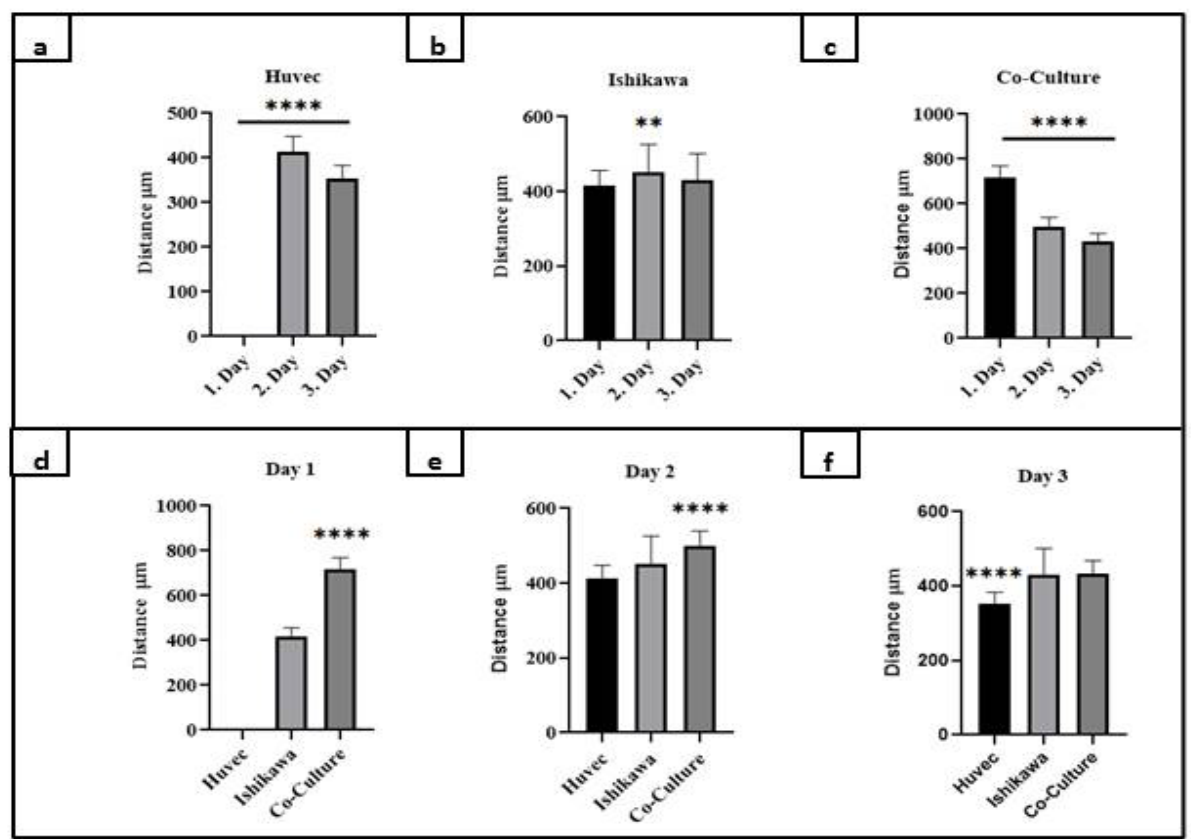

Figure 6. Statistical Analysis of mono-culture and co-culture Spheroids. (a) Daily changes of mean sizes of Huvec mono-culture spheroids $(1 \mathrm{st}-2 \mathrm{nd}-3 \mathrm{rd}$. days $* * * * \mathrm{p}<0,0001)$. (b) Daily changes of mean sizes of Ishikawa monoculture spheroids (2nd day $* * p=0,0010)$. (c) Daily changes of mean sizes of co-culture spheroids (1st-2nd-3rd days $\mathrm{p}<0,0001$ ). (d) Comparison between first day sizes of Huvec and Ishikawa spheroids in mono-culture and co-culture system $(* * * * p<0,0001)$. (e) Comparison between second day sizes of Huvec and Ishikawa spheroids in mono-culture and co-culture system $(* * * * p<0,0001)$. (f) Comparison between third day sizes of Huvec and Ishikawa spheroids in mono-culture and co-culture system $(* * * * \mathrm{p}<0,0001)$.

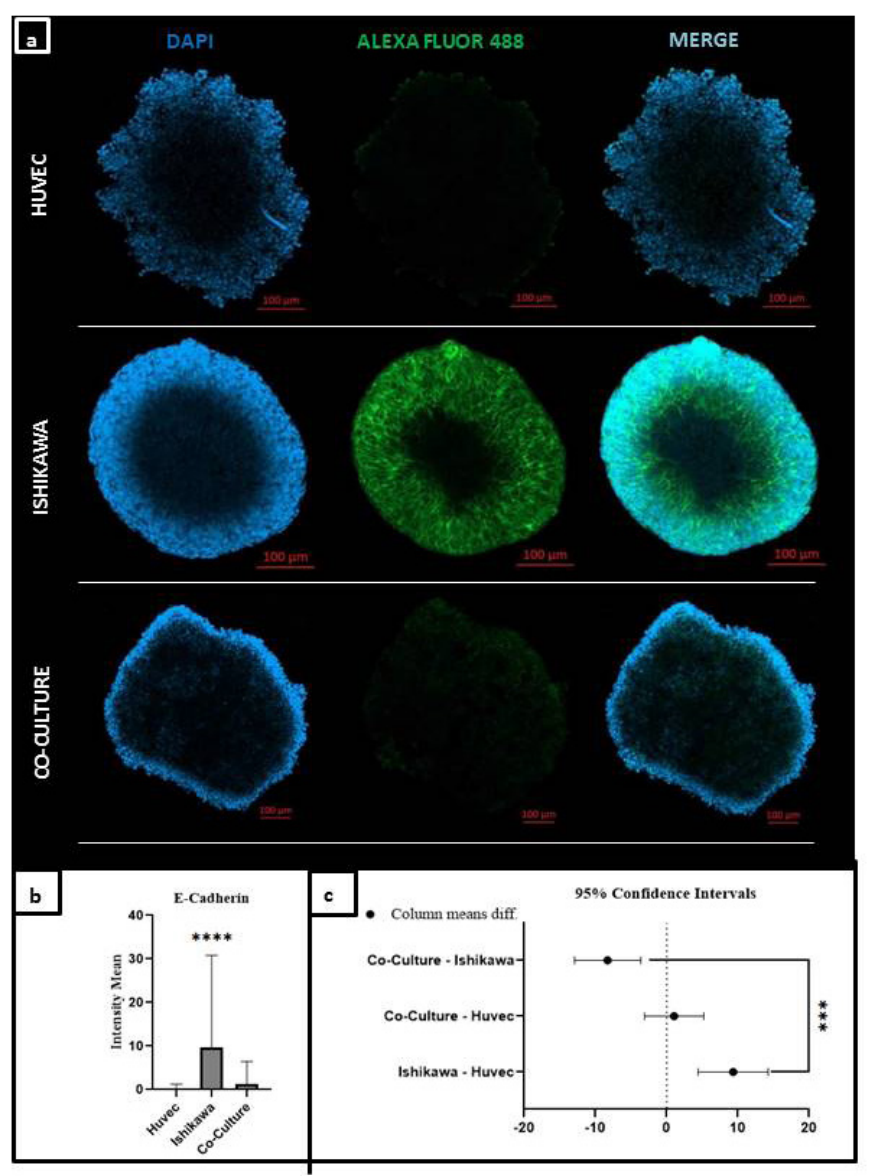

Figure 7. Immunofluorescence Staining of E-cadherin Performed Using Alexa Fluor 488 (membrane of cells) and Dapi (nuclei). (a) Images of Huvec and Ishikawa cells in mono-culture and co-culture models (Scale bar $=100 \mu \mathrm{m}$ ). (b) Mean E-cadherin expression levels of Huvec and Ishikawa spheroids in mono-culture and co-culture models. (c) Statistical analysis of mean E-cadherin levels of spheroids in mono-culture and co-culture models. (Bonferroni*** $\mathrm{p}<0,0001)$. 


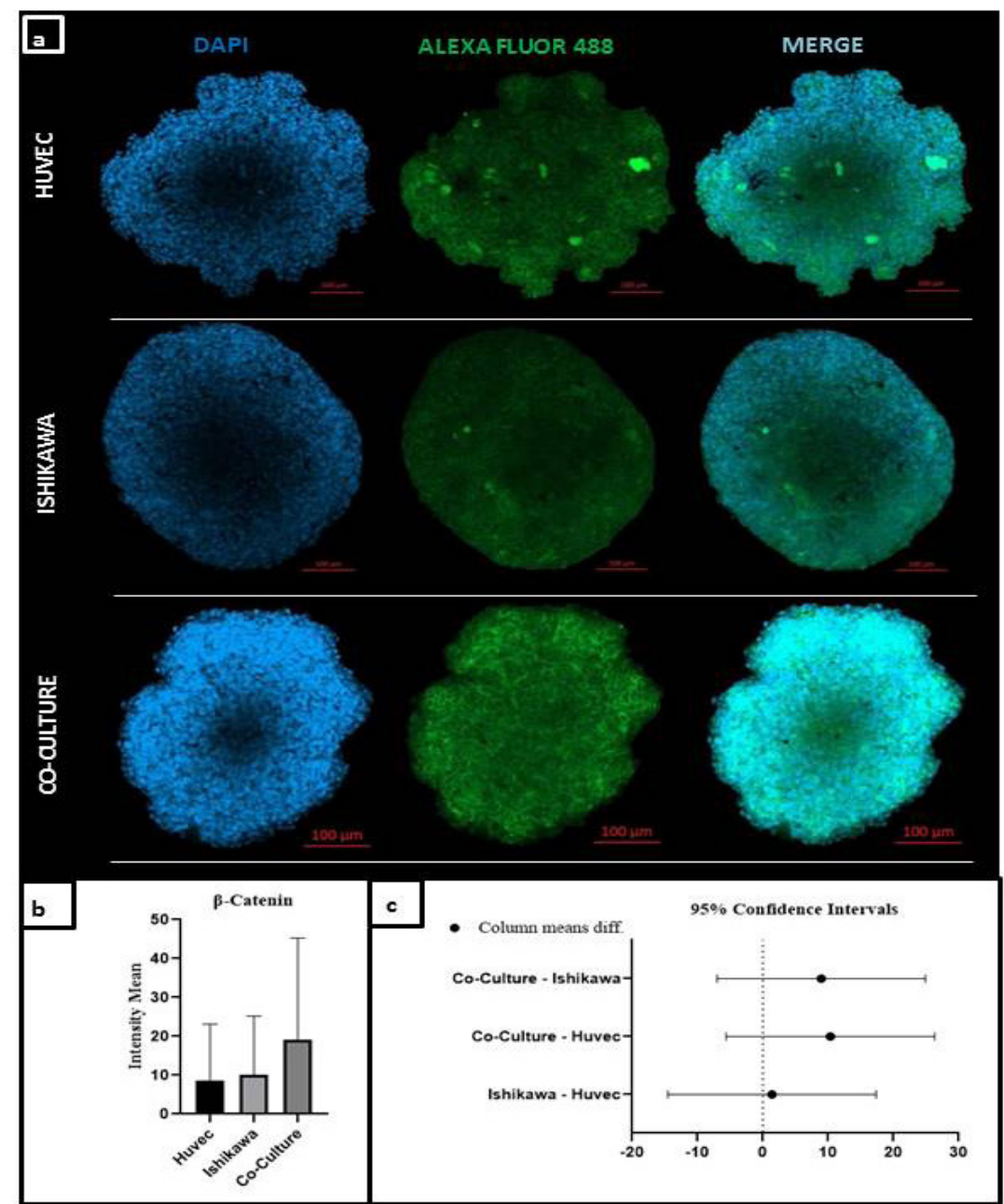

Figure 8. Immunofluorescence Staining of $\beta$-catenin Performed Using Alexa Fluor 488 (membrane of cells) and Dapi (nuclei). (a) Images of Huvec and Ishikawa cells in mono-culture and co-culture models (Scale bar $=100 \mu \mathrm{m}$ ). (b) Mean $\beta$-catenin expression levels of Huvec and Ishikawa spheroids in mono-culture and co-culture models. (c) Statistical analysis of mean $\beta$-catenin levels of spheroids in mono-culture and co-culture models. (Tukey $\mathrm{p}>0.05$ ).

conditions they do not express E-cadherin. Endothelium expresses VE-cadherin and $\mathrm{N}$-cadherin. A region of $\mathrm{N}$-cadherin is responsible for the activation of receptors of the fibroblast growth factor (FGF) in the endothelial cell (Bazzoni and Dejana, 2004). FGF-1 receptor-mediated bFGF, found in Huvec cells, decreases the expression of E-Cadherin (Wu et al., 2008). As in these studies, it is thought that the decreased expression of E-cadherin in co-culture spheroids is associated with suppression of E-cadherin expression in endothelial cells. The endothelial cells we used as stromal cells may have decreased the E-cadherin expression of Ishikawa cells.

$\beta$-catenin is responsible for maintaining cell polarity by interacting with E-cadherin, a critical regulator of cell-cell adhesion in the plasma membrane (Wang et al., 2015). $\beta$-catenin binds to the cytoplasmic extension of the E-cadherin mostly at adhesion junctions in normal epithelial cells (Pálmer et al., 2001). It has been previously observed that $\beta$-catenin expression in pancreatic cancer cells increases in the presence of stromal cells (Froeling et al., 2009). However, no increase in $\beta$-catenin expression in co-culture spheroids was observed in this study. Several studies have shown that $\beta$-catenin is a key modulator of cancer cell proliferation and survival. $\beta$-catenin has also been found to support tumor growth by promoting angiogenesis in cancers (Thakur and Mishra, 2013). It was observed that $\beta$-catenin expression increased significantly in two dimensional Ishikawa cells compared to the other two groups.

Changes in expression of E-cadherin in the three dimensional and two dimensional co-culture models suggest that in vivo cells are in a more complex microenvironment and there are differences in signaling mechanisms. Therefore, three dimensional co-culture techniques could be more applicable for future research.

In addition, the biggest problem encountered in cell culture studies by forming three dimensional spheroids is that the standardization of size (Weiswald et al., 2015). Although the number of cells seeded while forming spheroids is equal, the sizes of the resulting spheroids cannot be controlled. This problem can be avoided by producing a large number of spheroids and selecting the ones as equal to each other as possible.

In our study, the number of cells we used to form spheroids and the days we followed were indicated. We believe that this will provide convenience for other researchers who will work with the spheroids formed with Ishikawa and Huvec cells from now on.

Asian Pacific Journal of Cancer Prevention, Vol 23 


\section{Author Contribution Statement}

The authors confirm contribution to the paper as follows: study conception and design: Mervenur Kalender, Muhammet Volkan Bulbul and İlknur Keskin; data collection: Mervenur Kalender, Muhammet Volkan Bulbul; analysis and interpretation of results: Mervenur Kalender, Muhammet Volkan Bulbul; draft manuscript preparation: Mervenur Kalender, Muhammet Volkan Bulbul, Bircan Kolbasi and. All authors reviewed the results and approved the final version of the manuscript.

\section{Acknowledgements}

This study was carried out with the support of the infrastructure provided by Istanbul Medipol University Health Sciences and Technologies Research Institute (SABITA). This research did not receive any specific grant from funding agencies in the public, commercial, or not-for-profit sectors.

The authors declare that they have no known competing financial interests or personal relationships that could have appeared to influence the work reported in this paper.

\section{References}

Bazzoni G, Dejana E (2004). Endothelial cell-to-cell junctions: molecular organization and role in vascular homeostasis. Physiol Rev, 84, 869-901.

Betriu N, Semino CE (2018). Development of a 3D co-culture system as a cancer model using a self-assembling peptide scaffold. Gels, 4, 65.

Birgersdotter A, Sandberg R, Ernberg I. Gene expression perturbation in vitro - a growing case for three-dimensional (3D) culture systems. Sem Cancer Biol, 2005, 405-12.

Breslin S, O'Driscoll L (2016). The relevance of using 3D cell cultures, in addition to $2 \mathrm{D}$ monolayer cultures, when evaluating breast cancer drug sensitivity and resistance. Oncotarget, 7, 45745.

Chao Y, Wu Q, Shepard C, et al (2012). Hepatocyte induced reexpression of E-cadherin in breast and prostate cancer cells increases chemoresistance. Clin Exp Metastasis, 29, 39-50.

Duval K, Grover H, Han L-H, et al (2017). Modeling physiological events in 2D vs. 3D cell culture. Physiology, 32, 266-77.

Francavilla C, Maddaluno L, Cavallaro U (2009). The functional role of cell adhesion molecules in tumor angiogenesis. Sem Cancer Biol, 2009, 298-309.

Froeling FE, Mirza TA, Feakins RM, et al (2009). Organotypic culture model of pancreatic cancer demonstrates that stromal cells modulate E-cadherin, $\beta$-catenin, and Ezrin expression in tumor cells. Am J Pathol, 175, 636-48.

Ghasemi A, Saeidi J, Azimi-Nejad M, et al (2019). Leptininduced signaling pathways in cancer cell migration and invasion. Cell Oncol, 2019, 1-18.

Hess MW, Pfaller K, Ebner HL, et al (2010). 3D versus 2D cell culture: implications for electron microscopy. In 'Methods in cell biology', Eds Elsevier, pp 649-70.

Huh D, Hamilton GA, Ingber DE (2011). From 3D cell culture to organs-on-chips. Trends Cell Biol, 21, 745-54.

Iglesias JM, Beloqui I, Garcia-Garcia F, et al (2013). Mammosphere formation in breast carcinoma cell lines depends upon expression of E-cadherin. PloS one, 8, e77281.

Im G-I (2014). Coculture in musculoskeletal tissue regeneration.
Tissue Eng Part B: Reviews, 20, 545-54.

Kapałczyńska M, Kolenda T, Przybyła W, et al (2018). 2D and 3D cell cultures-a comparison of different types of cancer cell cultures. Arch Med Sci, 14, 910.

Khodarev NN, Yu J, Labay E, et al (2003). Tumour-endothelium interactions in co-culture: coordinated changes of gene expression profiles and phenotypic properties of endothelial cells. J Cell Sci, 116, 1013-22.

Kim BB, Tae JY, Ko Y, et al (2019a). Lovastatin increases the proliferation and osteoblastic differentiation of human gingiva-derived stem cells in three-dimensional cultures. Exp Ther Med, 18, 3425-30.

Kim MJ, Chi BH, Yoo JJ, et al (2019b). Structure establishment of three-dimensional (3D) cell culture printing model for bladder cancer. PloS one, 14, e 0223689.

Knight E, Przyborski S (2015). Advances in 3D cell culture technologies enabling tissue-like structures to be created in vitro. J Anat, 227, 746-56.

Koledova Z (2017). 3D cell culture: an introduction. In '3D Cell Culture', Eds Springer, pp 1-11.

Lee J, Cuddihy MJ, Kotov NA (2008). Three-dimensional cell culture matrices: state of the art. Tissue Eng Part B: Reviews, 14, 61-86.

Liang W, Ferrara N (2016). The complex role of neutrophils in tumor angiogenesis and metastasis. Cancer Immunol Res, 4, 83-91.

Lin RZ, Chang HY (2008). Recent advances in three-dimensional multicellular spheroid culture for biomedical research. Biotechnol J, 3, 1172-84.

Marino S, Bishop RT, de Ridder D, et al (2019). 2D and 3D in vitro co-culture for cancer and bone cell interaction studies. In 'Bone Research Protocols', Eds Springer, pp 71-98.

Osman A, Afify SM, Hassan G, et al (2020). Revisiting cancer stem cells as the origin of cancer-associated cells in the tumor microenvironment: A Hypothetical View from the Potential of iPSCs. Cancers, 12, 879.

Pálmer HG, González-Sancho JM, Espada J, et al (2001). Vitamin D3 promotes the differentiation of colon carcinoma cells by the induction of E-cadherin and the inhibition of $\beta$-catenin signaling. $J$ Cell Biol, 154, 369-88.

Saggioro M, D'Angelo E, Bisogno G, et al (2020). Carcinoma and sarcoma microenvironment at a glance: Where We Are. Front Oncol, 10.

Schmidt EP, Kuebler WM, Lee WL, et al (2011). Adhesion molecules: master controllers of the circulatory system. Compr Physiol, 2011, 945-73.

Sobierajska K, Ciszewski WM, Sacewicz-Hofman I, et al (2020). Endothelial Cells in the Tumor Microenvironment. In 'Tumor Microenvironment', Eds Springer, pp 71-86.

Sousa B, Pereira J, Paredes J (2019). The crosstalk between cell adhesion and cancer metabolism. Int J Mol Sci, 20, 1933.

Stockmann C, Schadendorf D, Klose R, et al (2014). The impact of the immune system on tumor: angiogenesis and vascular remodeling. Front Oncol, 4, 69.

Sun T, Jackson S, Haycock JW, et al (2006). Culture of skin cells in $3 \mathrm{D}$ rather than $2 \mathrm{D}$ improves their ability to survive exposure to cytotoxic agents. $J$ Biotech, 122, 372-81.

Swaminathan S, Cranston AN, Clyne AM (2019). A threedimensional in vitro coculture model to quantify breast epithelial cell adhesion to endothelial cells. Tissue Eng Part C: Methods, 25, 609-18.

Takayama T, Shiozaki H, Shibamoto Sl, et al (1996). Betacatenin expression in human cancers. Am J Pathol, 148, 39.

Thakur R, Mishra DP (2013). Pharmacological modulation of beta-catenin and its applications in cancer therapy. J Cell Mol Med, 17, 449-56.

von Lersner A, Droesen L, Zijlstra A (2019). Modulation of 
cell adhesion and migration through regulation of the immunoglobulin superfamily member ALCAM/CD166. Clin Exp Metastasis, 36, 87-95.

Wang Z, Zhang H, Hou J, et al (2015). Clinical implications of $\beta$-catenin protein expression in breast cancer. Int J Clin Exp Pathol, 8, 14989.

Weiswald L-B, Bellet D, Dangles-Marie V (2015). Spherical cancer models in tumor biology. Neoplasia, 17, 1-15.

Wu J-C, Yan H-C, Chen W-T, et al (2008). JNK signaling pathway is required for bFGF-mediated surface cadherin downregulation on HUVEC. Exp Cell Res, 314, 421-9.

Yildiz-Ozturk E, Gulce-Iz S, Anil M, et al (2017). Cytotoxic responses of carnosic acid and doxorubicin on breast cancer cells in butterfly-shaped microchips in comparison to 2D and 3D culture. Cytotechnology, 69, 337-47.

Zheng X, Du X-L, Jiang T (2015). Prognostic significance of reduced immunohistochemical expression of E-cadherin in endometrial cancer-results of a meta-analysis. Int J Clin Exp Med, 8, 18689.

Zurina I, Shpichka A, Saburina I, et al (2018). 2D/3D buccal epithelial cell self-assembling as a tool for cell phenotype maintenance and fabrication of multilayered epithelial linings in vitro. Biomed Mater, 13, 054104.

\section{(c) (i) (8)}

This work is licensed under a Creative Commons AttributionNon Commercial 4.0 International License. 